\title{
Medicamentos e Controle de dor: Experiência de um Centro de Referência em Cuidados Paliativos no Brasil
}

doi: https://doi.org/10.32635/2176-9745.RBC.2019v65n2.365

\author{
Medication and Pain Control: Experience of a Brazilian Palliative Care Referral Center \\ Medicamentos y Control del Dolor: Experiencia de un Centro de Referencia en Cuidados Paliativos en Brasil
}

Simone Garruth dos Santos Machado Sampaio'; 'Luciana Branco da Motta²; Celia Pereira Caldas ${ }^{3}$

Resumo

Introduçáo: A dor é um dos sintomas mais prevalentes em pacientes com câncer, especialmente no último ano de vida, sendo frequente o controle inadequado. Objetivo: Delinear o perfil de medicamentos utilizados para controle de dor em uma unidade hospitalar de cuidados paliativos oncológicos. Método: Estudo transversal com acompanhamento longitudinal de todos os pacientes internados entre setembro e novembro de 2016. Escala Verbal Numérica (EVN), funcionalidade e medicamentos com potencial analgésico utilizados foram coletados por revisão de prontuário. Resultados: Os 399 pacientes internados compuseram 461 episódios de internação, sendo 429 (93\%) com o sintoma dor (controlada ou não). A idade média foi 62 anos, oito dias em média de internação, motivada por dor em $18 \%$ dos casos; e, em 35\%, o sintoma dor não estava controlado. Destes, a EVN foi quantificada como zero após dois dias em média. Segundo a escada analgésica, 29\% estavam no primeiro degrau, 11\% no segundo e $82 \%$ no terceiro. O uso do analgésico comum e de adjuvante foi verificado em mais de $80 \%$ dos episódios. A dose média equivalente de morfina oral foi $117 \mathrm{mg} /$ dia. Discussáo: O controle de dor observado foi superior e mais precoce se comparado com outros trabalhos semelhantes. A dose média de opioide (equipotência analgésica) foi semelhante à observada em outros estudos. Entretanto, o uso de opioide forte e de adjuvantes foi mais frequente do que o notado em outros serviços. Essa diferença pode justificar o melhor controle álgico. Conclusáo: A ação da equipe especializada proporciona melhor controle de sintomas.

Palavras-chave: Tratamento Farmacológico; Cuidados Paliativos; Manejo da dor; Dor do Câncer.

\section{Abstract}

Introduction: Pain is one of the most prevalent symptoms in cancer patients, especially in the last year of life, and its inadequate control is frequent. Objective: Outline the profile of drugs used for pain control in an exclusive oncology palliative care hospital unit. Method: A crosssectional study with longitudinal follow-up of all hospitalized patients between September and November 2016. Numeric Rating Scale (NRS), functionality and drugs with analgesic potential used were collected through medical chart review. Results: The 399 hospitalized patients presented 461 hospitalization episodes, of which 429 (93\%) were patients with pain symptom (controlled or not). The mean age was 62 years, with an average of 8 days of hospitalization motivated by pain in $18 \%$ of the cases and in $35 \%$, the pain symptom was not controlled. Of these, NRS was quantified as zero after 2 days in average. According to the Analgesic Ladder, 29\% were in the $1^{\text {st }}$ step, $11 \%$ in the second and $82 \%$ in the third. The use of common analgesic and of adjuvant was verified in more than $80 \%$ of the episodes. The mean equivalent dose of oral morphine was $117 \mathrm{mg} /$ day. Discussion: Pain control observed was higher and earlier when compared to other similar works. The mean opioid dose (analgesic equipotent) was analogous to the observed in other studies. However, the use of strong opioids and adjuvants was more frequent than what was noticed in other services. This difference may justify the better pain control. Conclusion: The action of skilled team grants better symptom control.

Key words: Drug Therapy; Palliative Care; Pain Management; Cancer Pain.

\section{Resumen}

Introducción: El dolor es uno de los síntomas más frecuentes en pacientes con cáncer, especialmente en el último año de vida, y el control inadecuado es frecuente. Objetivo: Delinear el perfil de los medicamentos utilizados para el control del dolor en una unidad hospitalaria de cuidados paliativos para el cáncer. Método: Estudio transversal con seguimiento longitudinal de todos los pacientes hospitalizados entre septiembre y noviembre de 2016. La Escala Numérica Verbal (EVN), la funcionalidad y los medicamentos con potencial analgésico utilizados se recogieron mediante revisión de registros médicos. Resultados: Los 399 pacientes hospitalizados constituyeron 461 episodios de hospitalización, de los cuales 429 (93\%) tuvieron dolor de síntomas (controlado o no). La edad promedio fue de 62 años, 8 días en promedio. La hospitalización fue motivada por dolor en el $18 \%$ de los casos y en $35 \%$ el síntoma de dolor no fue controlado. De estos, EVN se cuantificó como cero después de 2 días en promedio. Según la Escalera Analgésica, el $29 \%$ estaba en el primer paso, el $11 \%$ en el segundo y el $82 \%$ en el tercero. El uso de analgésicos y adyuvantes comunes se observó en más del 80\% de los episodios. La dosis equivalente promedio de morfina oral fue de 117 mg/día. Discusión: El control del dolor observado fue superior y anterior en comparación con otros trabajos similares. La dosis promedio de opioide (equipamiento analgésico) fue similar a la observada en otros estudios. Sin embargo, el uso de opioides y adyuvantes fuertes fue más frecuente que en otros servicios. Esta diferencia puede justificar el mejor control del dolor observado. Conclusión: La acción del equipo de expertos proporciona un mejor control de los síntomas.

Palavras clave: Tratamiento Farmacológico; Cuidados Paliativos; Manejo del Dolor; Dolor em Câncer.

\footnotetext{
1 Unidade de Cuidados Paliativos do Hospital do Câncer IV (HC IV) do Instituto Nacional de Câncer José Alencar Gomes da Silva (INCA). Rio de Janeiro (RJ), Brasil. Orcid iD: https://orcid.org/0000-0001-5537-7399

${ }^{2}$ Núcleo de Atenção ao Idoso (NAI) da Universidade do Estado do Rio de Janeiro (Uerj). Rio de Janeiro (RJ), Brasil. Orcid iD: https://orcid.org/0000-0001-9959-9719

${ }^{3} \mathrm{NAl} /$ Uerj. Rio de Janeiro (RJ), Brasil. Orcid iD: https://orcid.org/0000-0001-6903-1778

Endereço para correspondência: Simone Garruth dos Santos Machado Sampaio. Rua Visconde de Santa Isabel, 274 - Vila Izabel. Rio de Janeiro (RJ), Brasil. CEP 20560-120

E-mail: simonegarruth@gmail.com
} 


\section{INTRODUÇÃO}

A dor é um sintoma presente em $80 \%$ dos pacientes com câncer no último ano de vida e classificada como intensa em um quarto destes ${ }^{1}$.

Em 1986, a Organização Mundial da Saúde (OMS) lançou seu primeiro protocolo sobre o manejo da dor oncológica baseado na "escada analgésica". A estratégia era avaliar e adaptar as medicaçóes utilizadas conforme a necessidade do paciente em cada momento, de forma sequencial e progressiva. Estima-se que o alívio do sintoma possa ser alcançado em $70 \%$ a $90 \%$ dos casos com o método proposto ${ }^{2-5}$.

Apesar dos esforços da OMS, não houve redução da prevalência da dor se comparados os períodos de 19652004 a $2005-2014^{6}$.

Embora o tratamento analgésico esteja disponível para $70 \%$ a $90 \%$ dos pacientes com câncer, em $40 \%$ a $50 \%$ dos casos ele é inadequado ${ }^{7,8}$. Há vários relatos na literatura sobre controle inadequado da dor em pacientes oncológicos?.

São bem documentados a importância e os resultados positivos frutos da ação de uma equipe especialista em cuidados paliativos no controle de sintomas, entre eles, a dor. É importante que cada serviço conheça seus indicadores e busque estratégias para aprimorar o serviço ${ }^{10,11}$.

O presente trabalho é uma das etapas de um estudo para avaliação do tratamento da dor em pacientes em cuidados paliativos oncológico atendidos em um centro de referência. $\mathrm{O}$ objetivo deste estudo foi delinear o perfil de medicamentos utilizados para controle de dor em uma unidade hospitalar de cuidados paliativos oncológicos.

\section{MÉTODO}

Trata-se de um estudo transversal de caráter prospectivo no qual todos os pacientes admitidos para internação na unidade entre 1/9/2016 e 30/11/2016 foram acompanhados longitudinalmente ao longo da internação, por meio de revisão de prontuário.

O trabalho foi desenvolvido em um hospital público de cuidados paliativos oncológicos, considerado um centro de referência nacional, localizado na cidade do Rio de Janeiro, Brasil. Na unidade, são atendidos apenas pacientes com câncer sem possibilidade de tratamento oncológico específico.

Para a quantificação objetiva da dor e da funcionalidade, foram utilizadas escalas amplamente utilizadas na literatura mundial e no cenário da pesquisa.

A dor foi quantificada por intermédio da Escala Verbal Numérica (EVN), obtida solicitando ao paciente que atribua uma nota de 0 a 10 para a sua dor, sendo "0" ausência de dor e "10" a dor mais intensa possível de imaginar ${ }^{12-15}$.

A funcionalidade dos pacientes foi documentada pela Escala Karnofsky Performance Status (KPS) que consiste em uma escala numérica com 11 opções de classificação, variando de $100 \%$ (nenhuma queixa, ausência de evidência da doença) a $0 \%$ (morte) $^{16}$.

Foram obtidos dados sociodemográficos sobre a doença oncológica, motivo da internação, KPS e EVN na admissão e diariamente nas evoluçôes de rotina, e medicamentos administrados com potencial analgésico (de horário e resgate).

Foram considerados "com dor" todos os pacientes que usaram algum analgésico comum (dipirona, paracetamol) ou opioide, mesmo com EVN zero. A dor foi considerada controlada quando a EVN era zero. Registro "sem dor" foi considerado como EVN zero. Uso de opioide ou antitérmico exclusivamente para dispneia ou febre foram desconsiderados. Os medicamentos com potencial analgésico ${ }^{5,17-19}$ padronizados na unidade na ocasião da coleta eram: dipirona e paracetamol (analgésico comum); diclofenaco e tenoxican (anti-inflamatório não hormonal); codeína e tramadol (opioide fraco); morfina, metadona, oxicodona e fentanil transdérmico (opioide forte); amitriptilina, sertralina, citalopram e venlafaxina (antidepressivo); gabapentina e pregabalina (anticonvulsivante); dexametasona e prednisona (corticoesteroide); baclofeno (GABA agonista); haloperidol e risperidona (neuroléptico); pamidronato e zolendronato (bisfosfonato); cetamina; e lidocaína venosa.

Entre aqueles que se internaram com dor não controlada, foi calculado o tempo necessário (em dias) para o controle do sintoma (EVN zero). As internaçôes foram classificadas segundo a escada analgésica da OMS conforme o uso de analgésicos comuns ou opioides. A dose dos opioides foi convertida para morfina oral ${ }^{20}$.

Para fins de análise, a unidade básica considerada foi a internação - chamada de episódio - e não o paciente. Foi realizada a análise descritiva das variáveis coletadas com determinação das frequências, medidas de tendência central e dispersão. As medidas de associação foram calculadas utilizando os testes qui-quadrado para variáveis categóricas, Mann-Whitney para as variáveis numéricas e Wilcoxon pareado para variáveis numéricas pareadas. Todas essas variáveis coletadas possuem distribuição não normal segundo o teste de Shapiro-Wilk. Foi utilizado o programa estatístico $\mathrm{R}$.

O projeto foi aprovado pela Comitê de Ética em Pesquisa do Instituto Nacional de Câncer José Alencar Gomes da Silva (INCA) e do Hospital Universitário Pedro 
Ernesto da Universidade do Estado do Rio de Janeiro (Uerj), sob o número de parecer 1.630.518, de 11/6/2016 (CAAE 54919016.4.0000.5274).

\section{RESULTADOS}

Entre 1/9/2016 e 30/11/2016, 399 pacientes foram internados na unidade perfazendo um total de 461 episódios de internaçóes, sendo 348 pacientes com um único episódio, 41 pacientes com dois episódios, nove pacientes com três episódios e um paciente com quatro episódios.

A maioria da população era do sexo feminino $(63,4 \%)$, idade entre 17 e 94 anos, com média de 62 anos (+/-4,5, mediana 62). Os sítios de tumor primário mais frequentes foram: mama (15\%), colo do útero $(13,3 \%)$ e pulmão $(11,3 \%)$. Na Tabela 1 , está descrita a população do estudo.

O tempo médio global de internação foi de 8,2 dias (+/-7,1). Em 429 episódios de internação (370 pacientes), foram utilizados medicamentos analgésicos e, por isso, os episódios classificados como "com dor" (Tabela 2). Em 32 episódios (29 pacientes), os pacientes não receberam medicações analgésicas ao longo de toda internação ou utilizaram morfina exclusivamente para dispneia e, consequentemente, foram classificados como "sem dor" e excluídos das análises posteriores.

Considerando a avaliação da dor no momento da admissão, em 144 episódios (33,6\%), a dor não estava controlada (EVN $>0)$. Destes, em 90 episódios $(62,5 \%)$, a dor foi documentada como controlada em até 24 horas após a internação. O tempo médio para o controle da dor foi de 2,1 dias (+/-1,9; IC 95\% 1,7-2,4), variando de zero a dez dias. Apenas um paciente não obteve controle da dor ao longo da internação.

Entre os 429 episódios com uso de analgésico, em 29 $(6,8 \%)$, a medicação utilizada correspondia ao primeiro degrau da escada analgésica; em 46 (10,7\%), ao segundo degrau; e, em 354 (82,5\%), ao terceiro degrau. Havia relato de uso de opioide antes da internação em 356 (83\%) episódios.

A associação com analgésico comum e adjuvantes nos pacientes classificados como segundo e terceiro degraus está descrita na Tabela 3. Em 351 episódios $(87,8 \%)$ com uso de opioide, o analgésico comum foi mantido.

O opioide mais utilizado foi a morfina, administrada por via endovenosa contínua em 41 episódios, por 2,6 dias em média (+/-1,7; variação de 1 a 8 dias). Os resgates de morfina (SOS) foram utilizados em 165 episódios, com média de 1,4 resgates por dia $(+/-0,7)$, variando de um a 15 resgates por dia. A utilizaçáo dos opioides com sua dose regular e como resgate está descrita na Tabela 4.

A dose média de opioide convertido em morfina oral foi 117,1 mg (IC 95\% 105,2-129,0). Houve aumento na dose média da morfina ao longo da internação. No
Tabela 1. Caracterização dos pacientes internados na unidade de cuidados paliativos oncológicos entre 1/9/2016 e 30/11/2016, quanto a características sociodemográficas, local do tumor e tratamento oncológico recebido

\begin{tabular}{|c|c|c|}
\hline & $\begin{array}{l}\text { Número } \\
\text { absoluto }\end{array}$ & $\%$ \\
\hline \multicolumn{3}{|l|}{ Sexo } \\
\hline Masculino & 146 & 36,6 \\
\hline Feminino & 253 & 63,4 \\
\hline \multicolumn{3}{|l|}{ Tumor primário } \\
\hline Aparelho ginecológico & 90 & 22,6 \\
\hline Aparelho digestório & 88 & 22,1 \\
\hline Mama & 60 & 15,0 \\
\hline Pulmão & 45 & 11,3 \\
\hline Cabeça e pescoço & 43 & 10,8 \\
\hline Pele & 19 & 4,8 \\
\hline Aparelho urinário & 19 & 4,8 \\
\hline Sarcoma & 13 & 3,3 \\
\hline Outros & 22 & 5,5 \\
\hline \multicolumn{3}{|c|}{ Locais de progressão de doença } \\
\hline Local & 293 & 73,4 \\
\hline Linfonodo & 188 & 47,1 \\
\hline Pulmão & 128 & 32,1 \\
\hline Osso & 94 & 23,6 \\
\hline Fígado & 84 & 21,1 \\
\hline Peritônio & 68 & 17,0 \\
\hline Sistema nervoso central & 60 & 15,0 \\
\hline Pleura & 49 & 12,3 \\
\hline Pele & 7 & 1,8 \\
\hline Adrenal & 6 & 1,5 \\
\hline Ovário & 5 & 1,3 \\
\hline Medula óssea & 3 & 0,8 \\
\hline Pâncreas & 1 & 0,3 \\
\hline Intravascular & 1 & 0,3 \\
\hline Outro & 1 & 0,3 \\
\hline \multicolumn{3}{|c|}{ Tratamento oncológico realizado } \\
\hline $\mathrm{RT}^{*}$ e $\mathrm{QT} \mathrm{T}^{* *}$ & 65 & 16,3 \\
\hline Cirurgia, RT e QT & 54 & 13,5 \\
\hline QT exclusiva & 49 & 12,3 \\
\hline Cirurgia e QT & 45 & 11,3 \\
\hline RT exclusiva & 37 & 9,3 \\
\hline Cirurgia exclusiva & 34 & 8,5 \\
\hline Cirurgia e RT & 32 & 8,0 \\
\hline $\mathrm{QT}, \mathrm{RT}$ e $\mathrm{BQT} \mathrm{T}^{* * *}$ & 21 & 5,3 \\
\hline Cirurgia, RT, BQT e QT & 4 & 1,0 \\
\hline RT e BQT & 2 & 0,5 \\
\hline $\begin{array}{l}\text { Sem tratamento } \\
\text { específico }\end{array}$ & 56 & 14,0 \\
\hline
\end{tabular}


Tabela 2. Descrição dos episódios de internação em que foram utilizadas medicações com potencial analgésico na unidade de cuidados paliativos oncológicos entre 1/9/2016 a 30/1 1/2016

\begin{tabular}{|c|c|c|}
\hline & \multicolumn{2}{|c|}{ Episódios com dor } \\
\hline & $\mathbf{n}$ & $\%$ \\
\hline $\begin{array}{l}\text { Número de } \\
\text { internações (episódios) }\end{array}$ & 429 & 93,1 \\
\hline \multicolumn{3}{|l|}{ Tempo de internação } \\
\hline Média & 8,4 & \\
\hline Desvio-padrão & 7,3 & \\
\hline Mínimo & 1 & \\
\hline $1^{\circ}$ quartil & 4 & \\
\hline Mediana & 6 & \\
\hline $3^{\circ}$ quartil & 11 & \\
\hline Máximo & 56 & \\
\hline \multicolumn{3}{|l|}{ Origem do paciente } \\
\hline Ambulatório & 72 & 16,8 \\
\hline $\begin{array}{l}\text { Serviço de Pronto } \\
\text { Atendimento }\end{array}$ & 260 & 60,6 \\
\hline Transferência interna & 95 & 22,1 \\
\hline Transferência externa & 2 & 0,5 \\
\hline \multicolumn{3}{|l|}{ Motivo da internação } \\
\hline Transferência & 87 & 20,3 \\
\hline Dor & 78 & 18,2 \\
\hline Dispneia & 59 & 13,8 \\
\hline Vômitos & 31 & 7,2 \\
\hline Desidratação & 26 & 6,1 \\
\hline Sonolência & 23 & 5,4 \\
\hline Delirium & 19 & 4,4 \\
\hline Infecção & 16 & 3,7 \\
\hline Sangramento & 15 & 3,5 \\
\hline Questões sociais & 11 & 2,6 \\
\hline Outros & 54 & 12,6 \\
\hline \multicolumn{3}{|l|}{ KPS na admissão } \\
\hline $10 \%$ & 6 & 1,4 \\
\hline $20 \%$ & 43 & 10 \\
\hline $30 \%$ & 187 & 43,6 \\
\hline $40 \%$ & 121 & 28,2 \\
\hline $50 \%$ & 50 & 11,7 \\
\hline $60 \%$ & 11 & 2,6 \\
\hline $70 \%$ & 4 & 0,9 \\
\hline $80 \%$ & 1 & 0,2 \\
\hline Sem informação & 6 & 1,4 \\
\hline \multicolumn{3}{|l|}{ KPS na alta } \\
\hline $20 \%$ & 4 & 2,7 \\
\hline $30 \%$ & 30 & 20,5 \\
\hline $40 \%$ & 57 & 39 \\
\hline $50 \%$ & 34 & 23,3 \\
\hline $60 \%$ & 9 & 6,2 \\
\hline $70 \%$ & 2 & 1,4 \\
\hline $80 \%$ & 0 & 0 \\
\hline Sem informação & 10 & 6,8 \\
\hline \multicolumn{3}{|l|}{ Desfecho do episódio } \\
\hline Alta & 146 & 34 \\
\hline Óbito & 283 & 66 \\
\hline
\end{tabular}

Legenda: KPS = Escala Karnofsky Performance Status. primeiro dia de internação, a média foi $94,1 \mathrm{mg}(+/-$ 105,3, IC 95\% 83,2-104,9) e, no último dia, 139,2 mg (+/-158,2 IC 95\% 123,2-155,3), com significância estatística (p-valor $<0,00)$.

A associação de medicamentos adjuvantes se mostrou eficaz para o controle precoce da dor. Com seu uso, a dor foi controlada em 0,8 dias $(+/-0,4$; IC $95 \% 0,6-1,1)$ versus 2,2 dias em média $(+/-2,0$; IC $95 \%$ 1,8-2,5) sem o uso destes. Mesmo excluindo o uso de corticoides e antipsicóticos, houve controle mais rápido da dor: 1,7 dias $(+/-1,4$; IC 95\% 1,3-2,0) versus 2,4 dias (+/-2,2; IC 95\% 0,9-2,9) em média, respectivamente. Essas associações foram estatisticamente significativas ( $\mathrm{p}$-valor $<0,00$ ).

Os adjuvantes utilizados estão descritos na Tabela 5. Os cálculos foram feitos considerando apenas os dias em que foram prescritos.

Em apenas 16 dos 323 episódios com uso de dexametasona, foi mencionada a indicação para dor. $\mathrm{Na}$ Tabela 5, foi incluída a dose global da droga.

A dor como indicação de uso do haloperidol foi mencionada em três dos 148 episódios, em oito dos 71 para o midazolam e em um dos 87 para o diazepam. Assim como para a dexametasona, a dor frequentemente era um dos motivos do uso.

Em nenhum caso, o uso de prednisona e de risperidona foi descrito como indicado para controle da dor. Em cinco episódios, havia prescrição do midazolam oral e parenteral, perfazendo um total de uso em 71 episódios com uso da substância.

O pamidronato foi prescrito com dose de $90 \mathrm{mg} \mathrm{em}$ 20 episódios, sendo quatro deles para controle da dor. O zolendronato foi utilizado na dose de $15 \mathrm{mg}$ em 24 episódios e, em 17 deles, a indicação era dor. Ambos os bisfosfonatos foram prescritos em um ciclo por episódio.

A dexmedetomidina foi utilizada em três episódios, sempre em infusão contínua com dose média $0,6 \mathrm{mcg} /$ $\mathrm{kg} / \mathrm{h}(+/-0,18)$, variando de 0,5 a $0,9 \mathrm{mcg} / \mathrm{kg} / \mathrm{h}$.

Não houve uso de cetamina e lidocaína venosa no período estudado.

Um paciente utilizou medicamentos não padronizados no hospital: duloxetina $60 \mathrm{mg} /$ dia e alprazolam $4 \mathrm{mg} / \mathrm{dia}$.

\section{DISCUSSÃO}

A população se assemelha à descrita por Lima et al. ${ }^{21}$ em uma enfermaria de oncologia de um hospital universitário do Nordeste do Brasil: predomínio de mulheres $(65 \%)$ e sítios primários mais comuns (mama $10 \%$ e colo do útero $15,2 \%$ ). Embora no referido trabalho o cenário estudado tenha sido uma enfermaria de oncologia (pacientes em tratamento oncológico específico e paliativo), 95\% estavam com estadiamento III ou IV. 
Tabela 3. Distribuição dos episódios de internação na unidade de cuidados paliativos oncológicos conforme a classificação pela escada analgésica e o uso de medicamentos adjuvantes, entre 1/9/2016 a 30/11/2016

\begin{tabular}{|c|c|c|c|c|c|c|}
\hline & \multicolumn{2}{|c|}{$\begin{array}{c}\text { Degrau } 1 \\
\text { (n 29) }\end{array}$} & \multicolumn{2}{|c|}{$\begin{array}{c}\text { Degrau } 2 \\
\text { (n 46) }\end{array}$} & \multicolumn{2}{|c|}{$\begin{array}{c}\text { Degrau } 3 \\
\text { (n } 354)\end{array}$} \\
\hline & $\mathbf{n}$ & $\%$ & $\mathbf{n}$ & $\%$ & $\mathbf{n}$ & $\%$ \\
\hline Uso de analgésico comum (dipirona ou paracetamol) & 29 & 100 & 41 & 89,1 & 310 & 87,6 \\
\hline Uso de adjuvante (qualquer classe) & 25 & 86,2 & 39 & 84,8 & 323 & 91,2 \\
\hline Antidepressivo & 0 & 0 & 7 & 15,2 & 59 & 16,7 \\
\hline Gabapentina ou pregabalina & 1 & 3,4 & 7 & 15,2 & 114 & 32,2 \\
\hline Baclofeno & 0 & 0 & 0 & 0 & 9 & 2,5 \\
\hline Neuroléptico & 11 & 37,9 & 13 & 28,2 & 132 & 37,3 \\
\hline Corticosteroide & 18 & 62,1 & 28 & 60,9 & 280 & 79,1 \\
\hline Uso de adjuvante (excluídos neuroléptico e corticosteroide) & 1 & 3,4 & 12 & 26,1 & 133 & 37,6 \\
\hline
\end{tabular}

Tabela 4. Dose diária de analgésico opioide prescrita na unidade de cuidados paliativos oncológicos entre 1/9/2016 e 30/1 1/2016

\begin{tabular}{l|c|ccc}
\hline \multicolumn{1}{c|}{ Analgésico opioide } & Frequência de uso* & $\begin{array}{c}\text { Média } \\
\text { (+/-desvio-padrão) }\end{array}$ & Mín. & Máx. \\
\hline Morfina regular oral (mg)** & 225 & $119,9(+/-111,7)$ & 18 & 800 \\
Morfina resgate oral (mg)*** & 137 & $38,4(+/-47,0)$ & 3 & 231 \\
Tramadol (mg) & 85 & $251,9(+/-103,4)$ & 50 & 400 \\
Codeina (mg) & 12 & $135(+/-41,5)$ & 90 & 240 \\
Oxicodona (mg) & 18 & $129,4(+/-97,0)$ & 20 & 320 \\
Fentanil transdérmico (mcg/h) & 21 & $68,5(+/-79,9)$ & 12 & 253 \\
Metadona oral $(\mathrm{mg})^{* *}$ & 51 & $34,5(+/-32,6)$ & 4 & 144 \\
\hline
\end{tabular}

Legendas: *Número de episódios em que foi prescrito o medicamento; **As prescriçôes por via parenteral foram convertidas para via oral.

Tabela 5. Descrição das doses diárias dos medicamentos analgésicos e adjuvantes prescritos na unidade de cuidados paliativos oncológicos entre $1 / 9 / 2016$ e 30/11/2016

\begin{tabular}{|c|c|c|c|c|c|c|}
\hline \multirow{2}{*}{ Medicamento } & \multirow{2}{*}{ Média } & \multirow{2}{*}{ Mediana } & \multirow{2}{*}{ Mín. } & \multirow{2}{*}{ Máx. } & \multicolumn{2}{|c|}{ Frequência de uso* } \\
\hline & & & & & n & $\%$ \\
\hline Dipirona (g) & 6,2 & 6,0 & 1,0 & 12,0 & 364 & $84,8 \%$ \\
\hline Paracetamol (g) & 3,0 & 3,0 & 3,0 & 3,0 & 2 & $0,5 \%$ \\
\hline Tenoxicam (mg) & 34,3 & 40,0 & 20,0 & 40,0 & 7 & $1,6 \%$ \\
\hline Amitriptilina (mg) & 35,3 & 25,0 & 25,0 & 75,0 & 26 & $6,1 \%$ \\
\hline Sertralina (mg) & 48,4 & 50,0 & 25,0 & 77,0 & 9 & $2,1 \%$ \\
\hline Citalopram (mg) & 19,8 & 20,0 & 15,7 & 20,0 & 24 & $5,6 \%$ \\
\hline Venlafaxina (mg) & 47,4 & 37,5 & 37,5 & 75,0 & 16 & $3,7 \%$ \\
\hline Baclofeno (mg) & 17,7 & 12,7 & 8,0 & 30,0 & 9 & $2,1 \%$ \\
\hline Gabapentina (mg) & $1.478,3$ & $1.200,0$ & 300,0 & $3.600,0$ & 105 & $24,5 \%$ \\
\hline Pregabalina (mg) & 124,8 & 150,0 & 75,0 & 212,5 & 18 & $4,2 \%$ \\
\hline Clonidina (mg) & 0,2 & 0,2 & 0,2 & 0,3 & 3 & $0,7 \%$ \\
\hline Dexametasona (mg) & 11,8 & 12,0 & 2,0 & 18,5 & 323 & $75,5 \%$ \\
\hline Prednisona (mg) & 25,0 & 25,0 & 10,0 & 40,0 & 4 & $0,9 \%$ \\
\hline Haloperidol (mg) & 5,9 & 5,0 & 0,5 & 16,3 & 148 & $34,5 \%$ \\
\hline Risperidona (mg) & 1,3 & 1,0 & 0,5 & 2,0 & 17 & $4,0 \%$ \\
\hline Midazolam parenteral (mg) & 12,4 & 5,0 & 2,0 & 103,5 & 55 & \\
\hline $12,8 \%$ & & & & & & \\
\hline Midazolam oral (mg) & 11,1 & 11,3 & 7,5 & 15,0 & 21 & $4,9 \%$ \\
\hline Diazepam (mg) & 7,8 & 6,0 & 2,5 & 20,0 & 87 & $20,3 \%$ \\
\hline
\end{tabular}

Legenda: *Número de episódios em que foi prescrito o medicamento. 
Conforme os critérios estabelecidos no presente estudo, a grande maioria da população (370 pacientes, $92,7 \%$ ) apresentava o sintoma "dor", correspondendo a 429 episódios de internação $(93,1 \%)$ no período estudado. Em apenas 35,2\% dos episódios, a dor náo estava controlada no momento da admissão. Desconsiderando a transferência hospitalar (onde o sintoma que levou à internação não foi identificado), a dor foi a principal causa de hospitalização (78 casos, 22,8\%). Semelhante foi a taxa de internação por dor encontrada em estudo na Dinamarca (20\%), embora tenha sido mencionada como presente em $70 \%$ dos casos de internação ${ }^{22}$.

O tempo médio para obter EVN zero foi de dois dias, tendo esse controle sido atingido em até 24 horas na maioria dos episódios. Esse achado foi superior ao descrito por outros autores. Lima et al. ${ }^{21}$ observaram $70 \%$ de dor sem controle ao internar e $15 \%$ ainda apresentavam dor no oitavo dia de internaçáo. $\mathrm{Na}$ Coreia, $17 \%$ não obtiveram dor controlada no sétimo dia de internação ${ }^{23}$. Em um estudo realizado na Tailândia, o tempo médio de controle da dor foi de sete dias e, em apenas $42 \%$ dos pacientes, foi descrito $\mathrm{EVN}=<4$ no sétimo dia de tratamento ${ }^{24}$. Neste último estudo, chama a atenção que a EVN média no último dia de internaçáo foi de 3,7 , superior à média observada no sétimo dia de internação $(2,3)$.

Duas diferenças metodológicas entre o presente estudo e os demais citados precisam ser destacadas. A primeira é o valor da EVN em que a dor foi definida como controlada: 0 no presente estudo, $=<3^{21},=<4^{23}$. A segunda diferença é que, no presente estudo, quando o paciente não era capaz de quantificar a dor, mas o profissional registrava ausência de queixa álgica, foi atribuída EVN 0. Nos demais estudos, esses pacientes foram excluídos.

Sendo o controle dos sintomas um dos princípios dos cuidados paliativos, no caso da dor, é desejável que seja obtido o mais breve possível. Dada a frequência do sintoma observada ao ser admitido na internação, um controle mais precoce da dor pode propiciar uma desospitalização mais precoce.

Ainda comparando o presente estudo com os achados de Lima et al. ${ }^{21}$, a proporção de uso de analgésicos comuns foi semelhante ( $89 \%$ e 90\%). Já o uso de opioides fortes foi discrepante ( $82 \%$ e $51 \%$ ), assim como o uso de adjuvantes (90\% e 31\%). Essas diferenças podem justificar o controle mais precoce da dor observada neste trabalho.

Considerando o uso de bisfosfonato independente da indicação, a frequência de uso é semelhante (10\% e 13\%). Por outro lado, se for levado em conta apenas o uso dos bisfosfonatos para dor, o percentual do atual estudo cai para $0,4 \%$. Esse dado não é discriminado no outro estudo.

Embora haja progressão de doença para osso em $23,6 \%$ dos pacientes, apenas em 21 episódios (4,9\%) foram utilizados bisfosfonatos para controle de dor. Algumas possíveis justificativas para essa discrepância são: paciente em uso regular do bisfosfonato com ciclo mensal ou trimestral com internação fora do período de administração da droga, KPS muito baixo ou expectativa de evolução a óbito breve (tornando o uso da medicação uma futilidade terapêutica), uma vez que objetivo é controle de sintomas ${ }^{25}$.

Relacionando o perfil medicamentoso no dia em que a dor foi considerada controlada com o trabalho de Wangnamthip et al..$^{24}$, o uso de opioides foi maior no presente estudo (93\% e $71 \%$ ), com menor uso de opioide fraco (17\% e 46\%). O atual estudo utilizou menos anti-inflamatório não hormonal (2\% e 23\%) e antidepressivos (19\% e 51\%). A diferença nesse perfil medicamentoso justifica o melhor controle da dor observado no presente estudo.

Um estudo realizado na Itália observou distribuição pela escada analgésica diferente do presente estudo: $17 \%$ no primeiro degrau, $63 \%$ no segundo e $20 \%$ no terceiro. A população apresentava KPS muito superior e a maioria dos pacientes não apresentava o sintoma $\operatorname{dor}^{26}$.

$\mathrm{O}$ uso de antidepressivo foi equivalente ao encontrado por Janberidze et al. ${ }^{26}$ (14,5\% e $14 \%$ respectivamente). O fato de $75 \%$ dos pacientes em uso de antidepressivo não preencherem critério de depressão pode indicar o uso dessa classe medicamentosa como adjuvante.

O benefício dos gabapentinoides no controle da dor é bem definido na literatura, seja em monoterapia seja como adjuvante $^{27}$. No presente estudo, em $29 \%$ dos episódios, foi prescrito gabapentina ou pregabalina, sendo mais frequente sua associaçấo com opioide forte (32\%). Não houve uso como monoterapia.

A dose diária média de morfina oral (opioides convertidos segundo equipotência analgésica) observada no presente trabalho $(117 \mathrm{mg} / \mathrm{dia})$ foi próxima ao valor de $120 \mathrm{mg} /$ dia no estudo de Lundorff et al. ${ }^{22}$ e inferior aos $177 \mathrm{mg} / \mathrm{dia}$ no de Janberidze et al. ${ }^{26} \mathrm{~A}$ frequência de uso de analgésico comum concomitante ao opioide também foi semelhante ao achados de Lundorff et al. ${ }^{22}$ (85 e 83\%) e superior aos de Janberidze et al. ${ }^{26}$ (28\%). A associação com adjuvantes (excluindo neuroléptico e corticosteroides) foi maior nesta pesquisa do que nas outras duas (34, 25 e 11\%).

Embora a associação de opioide, ansiolítico e antipsicótico seja temida no tratamento da população geral $^{28}$, é usada com frequência em pacientes em paliação para controle de sintomas, especialmente no cuidado de fim de vida ${ }^{29}$. Golčić et al. ${ }^{30}$ conduziram um estudo retrospectivo com 765 pacientes comparando a sobrevida de pacientes com as diferentes associações dessas classes medicamentosas em um hospice na Croácia. Observaram sobrevida maior no grupo que recebeu 
opioide, antipsicótico e ansiolítico simultaneamente do que nas outras combinaçóes. A dose média de haloperidol foi $4,5 \mathrm{mg} / \mathrm{dia}(0,2$ a $20 \mathrm{mg} / \mathrm{dia})$ e administrada a $15 \%$ dos pacientes. Midazolam foi utilizado por $26 \%$ dos pacientes com dose média de $15 \mathrm{mg} / \mathrm{dia}(7,5$ a $30 \mathrm{mg} / \mathrm{dia})$ e o diazepam em $21 \%$, na dose média de $10 \mathrm{mg} / \mathrm{dia}$ (2 a $45 \mathrm{mg} / \mathrm{dia})$. A dose equivalente de morfina oral média foi 129,3 mg/dia (+/-145,9, mediana $80 \mathrm{mg} / \mathrm{dia})$. As doses médias maiores encontradas por Golčić et al. ${ }^{30}$, quando comparadas ao presente estudo, podem ser justificadas por terem incluído apenas pacientes em fase final de vida.

Alguns medicamentos possuem múltiplas indicaçóes em cuidados paliativos. Por exemplo: corticosteroides também são prescritos para fadiga, hiporexia e edema tumoral ${ }^{31,32}$; neurolépticos para delirium ${ }^{33}$ e náuseas ${ }^{34}$; benzodiazepínicos para sedação paliativa ${ }^{35}$ e insônia ${ }^{36}$. A análise de sua importância no controle da dor neste estudo foi muito prejudicada. O pesquisador procurou identificar a indicação de uso dessas classes medicamentosas, mas as informaçôes contidas no prontuário não esclareceram a grande maioria dos casos. A menção da indicação para controle álgico apareceu em apenas 5\% dos casos para a dexametasona, $2 \%$ para o haloperidol, 11\% para o midazolam e 1\% para diazepam. Ficou claro pela análise dos prontuários que, em raros casos a dor, era a principal indicação, embora em diversos episódios fosse uma das indicaçôes.

$\mathrm{O}$ fato de a dose de dexametasona ter sido semelhante nos dois grupos (com e sem dor) e maior quando apenas dispneia estava relatada corrobora a hipótese anterior. A dose de midazolam, tanto oral quanto parenteral, foi maior quando apenas o sintoma dispneia estava presente. Como náo foi diferenciado o uso para o controle da dor, é possível que as maiores doses tenham sido motivadas por dispneia e delirium no grupo com o sintoma dor. Da mesma forma, as doses relativamente altas utilizadas de haloperidol devem ter sido motivadas por delirium (sintoma frequente, especialmente nos últimos dias de vida). O não detalhamento dessa informação impossibilita conclusões precisas.

A ação de uma equipe de cuidados paliativos treinada leva ao melhor controle de sintomas e qualidade de vida dos pacientes em paliação oncológica. $\mathrm{Na}$ revisão sistemática e metanálise conduzida por Kassianos et al. ${ }^{10}$, foram encontrados resultados positivos em todos os artigos analisados, com destaque para importância da equipe multiprofissional. As diferenças observadas no perfil medicamentoso podem refletir uma expertise dos profissionais prescritores da unidade, e consequente melhor controle de sintomas.

A maior limitação deste estudo foi a coleta de dados restrita ao prontuário. Um estudo complementar com a quantificação da dor coletada diretamente com o paciente e a identificação do tipo de dor ao invés da avaliação do prontuário deve ser conduzido com intuito de confirmar os resultados obtidos.

\section{CONCLUSÃO}

Este trabalho veio atender a uma necessidade de conhecer melhor o tratamento medicamentoso para dor executado na unidade de cuidados paliativos oncológico estudada. Com perfil populacional semelhante a outros estudos, apesar das diferenças metodológicas, a prática do uso de adjuvantes e o manejo seguro de opioides fortes parecem os fatores associados aos achados favoráveis. Treinamento continuado da equipe e vigilância de indicadores são importantes para aprimorar os serviços oferecidos na unidade.

\section{CONTRIBUIÇÕES}

Todos os autores participaram da concepção e delineamento do estudo, análise e interpretação dos dados, redação e revisão do manuscrito e aprovaram a versão final.

\section{DECLARAÇÃO DE CONFLITO DE INTERESSE}

Nada a declarar.

\section{FONTES DE FINANCIAMENTO}

Não há.

\section{REFERÊNCIAS}

1. Pidgeon T, Johnson CE, Currow D, et al. A survey of patients' experience of pain and other symptoms while receiving care from palliative care services. BMJ Support Palliat Care. 2016;6(3):315-22. doi: http://dx.doi. org/10.1136/bmjspcare-2014-000748

2. Ventafridda V, Tamburini M, Caraceni A, et al. A validation study of the WHO method for cancer pain relief. Cancer. 1987;59(4):850-6. doi: https://doi. org/10.1002/1097-0142(19870215)59:4<850::AIDCNCR2820590432>3.0.CO;2-1

3. Mercadante S, Fulfaro F. World Health Organization guidelines for cancer pain: a reappraisal. Ann Oncol. 2005;16(Suppl 4):132-5. doi: https://doi.org/10.1093/ annonc/mdi922

4. Vargas-Schaffer G. Is the WHO analgesic ladder still valid? Twenty-four years of experience. Can Fam Physician. 2010;56(6):514-7.

5. World Health Organization. National cancer control programmes: policies and managerial guidelines. 2nd ed. Geneva: WHO; 2002. 
6. Haumann J, Joosten EBA, Van den Beuken-van Everdingen MHJ. Pain prevalence in cancer patients: Status quo or opportunities for improvement? Curr Opin Support Palliat Care. 2017;11(2):99-104. doi: https:// doi.org/10.1097/SPC.0000000000000261

7. Deandrea S, Montanari M, Moja L, et al. Prevalence of undertreatment in cancer pain. A review of published literature. Ann Oncol. 2008;19(12):1985-91. doi: https://doi.org/10.1093/annonc/mdn419

8. Cipta AM, Pietras CJ, Weiss TE, et al. Cancer-related pain management in clinical oncology. J Community Support Oncol. 2015;13(10):347-55. doi: https://doi. org/10.12788/jcso.0173

9. Haozous EA, Knobf MT. "All my tears were gone": suffering and cancer pain in Southwest American Indians. J Pain Symptom Manage. 2013;45(6):1050-60. doi: https://doi.org/10.1016/j. jpainsymman.2012.06.001

10. Kassianos AP, Ioannou M, Koutsantoni M, et al. The impact of specialized palliative care on cancer patients' health-related quality of life: a systematic review and meta-analysis. Support Care Cancer. 2018;26(1):61-79. doi: https://doi.org/10.1007/s00520-017-3895-1

11. Sampaio SGSM, Motta LB, Caldas, CP. Valuebased medicine and palliative care: how do they converge? Expert Rev Pharmacoecon Outcomes Res. 2019;19(5):509-515. doi: https://doi.org/10.1080/147 37167.2019.1651645

12. Fortunato JGS, Furtado MS, Hirabae LFA, et al. Escalas de dor no paciente crítico: uma revisão integrativa. Rev Hosp Univ Pedro Ernesto. 2013;12(3):110-7. doi: https://doi.org/10.12957/rhupe.2013.7538

13. Hennemann-Krause L. Dor no fim da vida: avaliar para tratar. Rev Hosp Univ Pedro Ernesto. 2012;11(2):26-31.

14. Jeter K, Blackwell S, Burke L, et al. Cancer symptom scale preferences: does one size fit all? BMJ Support Palliat Care. 2018;8(2):198-203. doi: http://dx.doi. org/10.1136/bmjspcare-2015-001018

15. Hjermstad MJ, Fayers PM, Haugen DF, et al. Studies comparing numerical rating scales, verbal rating scales, and visual analogue scales for assessment of pain intensity in adults: a systematic literature review. J Pain Symptom Manage. 2011;41(6):1073-93. doi: https:// doi.org/10.1016/j.jpainsymman.2010.08.016

16. Karnofsky DA, Burchenal JH. The clinical evaluation of chemotherapeutic agents in cancer. In: Mac Leod CM, editor. Evaluation of Chemotherapeutic Agents. New York: Columbia Univ Press; 1949. p. 199-205.

17. Thomaz A. Dor oncológica: conceitualizaçáo e tratamento farmacológico. Onco\&. 2010 ago./set.:24-9.

18. Portenoy RK. Treatment of cancer pain. Lancet. 2011;377(9784):2236-47. doi: https://doi.org/10.1016/ S0140-6736(11)60236-5
19. Scarborough BM, Smith CB. Optimal pain management for patients with cancer in the modern era. CA Cancer J Clin. 2018;68(3):182-96. doi: https://doi.org/10.3322/ caac. 21453

20. National Comprehensive Cancer Network. Adult Cancer Pain: NCCN guidelines. Rockledge (PA): National Comprehensive Cancer Network; 2018.

21. Lima AD, Maia IO, Costa Jr I, et al. Avaliação da dor em pacientes oncológicos internados em um hospital escola do nordeste do Brasil. Rev Dor. 2013;14(4):267-71. doi: http://dx.doi.org/10.1590/S1806-00132013000400007

22. Lundorff L, Peuckmann V, Sjogren P. Pain management of opioid-treated cancer patients in hospital settings in Denmark. Acta Anaesthesiol Scand. 2008;52(1):137-42. doi: https://doi.org/10.1111/j.1399-6576.2007.01522.x

23. Shin DW, Hwang SS, Oh J, et al. Variations in pain management outcomes among palliative care centers and the impact of organizational factors. Cancer. 2012;118(22):5688-97. doi: https://doi.org/10.1002/ cncr. 26722

24. Wangnamthip S, Euasobhon P, Siriussawakul A, et al. Effective pain management for inpatients at Siriraj Hospital: a retrospective study. J Med Assoc Thai. 2016;99(5):565-71.

25. Albert RH. End-of-life care: managing common symptoms. Am Fam Physician. 2017;95(6):356-61.

26. Janberidze E, Hjermstad MJ, Brunelli C, et al. The use of antidepressants in patients with advanced cancer--results from an international multicentre study. Psychooncology. 2014;23(10):1096-102. doi: https://doi.org/10.1002/ pon. 3541

27. Jordan RI, Mulvey MR, Bennett MI. A critical appraisal of gabapentinoids for pain in cancer patients. Curr Opin Support Palliat Care. 2018;12(2):108-17. doi: https:// doi.org/10.1097/SPC.0000000000000337

28. Park TW, Saitz R, Ganoczy D, et al. Benzodiazepine prescribing patterns and deaths from drug overdose among US veterans receiving opioid analgesics: casecohort study. BMJ. 2015;350:h2698. doi: https://doi. org/10.1136/bmj.h2698

29. Morita T, Tsunoda J, Inoue $S$, et al. Effects of high dose opioids and sedatives on survival in terminally ill cancer patients. J Pain Symptom Manage. 2001;21(4):282-9. doi: https://doi.org/10.1016/ s0885-3924(01)00258-5

30. Golčić M, Dobrila-Dintinjana R, Golčić G, et al. The impact of combined use of opioids, antipsychotics, and anxiolytics on survival in the hospice setting. J Pain Symptom Manage. 2018;55(1):22-30. doi: https://doi. org/10.1016/j.jpainsymman.2017.08.004

31. Lundström SH, Fürst CJ. The use of corticosteroids in Swedish palliative care. Acta Oncol. 2006;45(4):430-7. doi: https://doi.org/10.1080/02841860500401167 
32. Denton A, Shaw J. Corticosteroid prescribing in palliative care settings: A retrospective analysis in New Zealand. BMC Palliat Care. 2014;13(1):7. doi: https://doi. org/10.1186/1472-684X-13-7

33. Schrijver EJM, de Graaf K, de Vries OJ, et al. Efficacy and safety of haloperidol for in-hospital delirium prevention and treatment: a systematic review of current evidence. Eur J Intern Med. 2016;27:14-23. doi: https://doi. org/10.1016/j.ejim.2015.10.012

34. Murray-Brown F, Dorman S. Haloperidol for the treatment of nausea and vomiting in palliative care patients. Cochrane Database Syst Rev. 2015;(11):CD006271. doi: https://doi.org/10.1002/14651858.CD006271.pub3

35. Maltoni M, Setola E. Palliative sedation in patients with cancer. Cancer Control. 2015;22(4):433-41. doi: https:// doi.org/10.1177/107327481502200409

36. Frase L, Nissen C, Riemann D, et al. Making sleep easier: pharmacological interventions for insomnia. Expert Opin Pharmacother. 2018;19(13):1465-73. doi: https://doi.or g/10.1080/14656566.2018.1511705

Recebido em 25/7/2019

Aprovado em 11/10/2019 